\title{
Making Materials That Hate Water to Love Water: The Transformative Power of Chemistry*
}

\author{
Richard N. Zare ${ }^{1}$
}

\begin{abstract}
A simple nonaqueous reaction scheme for transforming the surface of plastics from hydrophobic to hydrophilic is presented. The chemical modification is achieved by the base-catalyzed trans-esterification of polyethylene terephthalate (PET), which is a commonly used plastic. Its use in blood collection tubes is discussed. The surface modification is permanent, inexpensive, rapid, and does not release contaminants. It also causes no optical or mechanical distortion of the plastic. This work demonstrates the power of chemistry to transform common materials.
\end{abstract}

Keywords: Hydrophilic; Hydrophobic; Polyethylene Terephthalate.

What a pleasure to be with you this morning and to have this opportunity to talk to you about materials for tomorrow, really something about the transformative power of chemistry. And I'm going to pick one particular material, one that I hope many of you are familiar with. I'm going to talk about blood collection tubes. At first, this sounds not very exciting. How many people have ever had their blood taken? Let's have a show of hands. Huge numbers in our audience! I tell you in the United States - I only have the figures for the United States - 3 billion tubes are made each year in which we collect blood and analyze it. So it is one of the major ways of being medically examined. Yet, the story behind blood collection tubes has many twists and turns, as I will explain. Here is the story of how I became involved.

Dr. Raffick Bowen (Figure 1), who is head of the Stanford Hospital in-charge of drawing and analyzing blood, came to me and he told me "I got a problem. Help, can you do something? I used to be able to use glass for my blood collection tubes but I am no longer allowed to do that. Doctors and nurses keep dropping the glass blood collection tubes on the floor and infecting themselves. So now I am only permitted to use plastic, and the plastic I use is made of something called polyethylene terephthalate also simply called PET. But the plastic

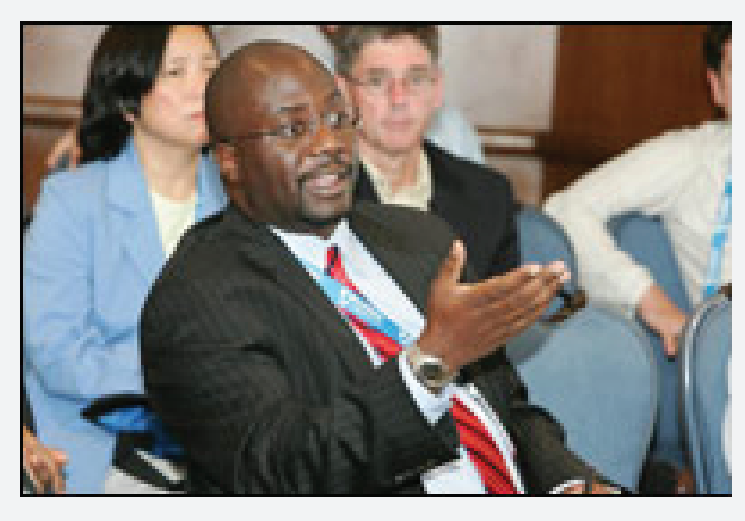

Figure 1. Dr. Raffick A. R. Bowen, Department of Pathology, Stanford University School of Medicine.

\footnotetext{
${ }^{1}$ Department of Chemistry, Stanford University, Stanford, California, USA. Published online 16 May 2017; doi:10.1142/S2529732517400028 *This article was transcribed from a presentation delivered by Professor Zare at the Molecular Frontiers Symposium, Chalmers University of Technology, Gothenburg, November 2014. It is published here in MFJ with permission from Professor Zare.

This is an Open Access article published by World Scientific Publishing Company. It is distributed under the terms of the Creative Commons Attribution 4.0 (CC-BY) License. Further distribution of this work is permitted, provided the original work is properly cited.
} 


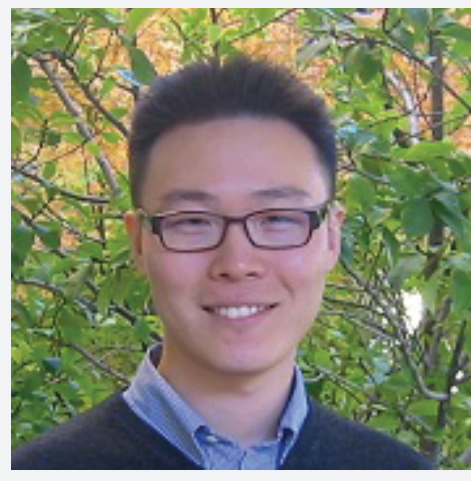

Figure 2. Dr. Samuel L. Kim, my former graduate student, who is presently at UCSF Medical School. pulls molecules out of our blood samples and interferes with our blood analysis. Can't you make the plastic act like glass?"

I was surprised I was asked as I am not known for my prowess in chemical synthesis. I did have a reputation of being willing to pursue problems outside my field of expertise. I did remember some organic chemistry from long ago, and I was able to convince a graduate student of mine, Samuel L. Kim (Figure 2), to give this problem a try, and very quickly something worked.

I'm going to tell you about how to change the surface of blood collection tubes that are plastic, from hating water to loving water, that is, to transform the surface from hydrophobic to hydrophilic. I'll go through how this is done.

Let's begin with the importance of this topic. It turns out blood collection and processing are the two major pre-analytical steps in clinical laboratory testing. If you look into where the errors in blood analysis occur, often it is caused by the blood collection tube, as revealed by a brief history of this topic. At first, people started using reusable glass syringes with steel hypodermic needles. Those were the first devices. And the glass syringes were expensive to manufacture and they could easily break. What ultimately prompted the replacement, however, was not that fact, but rather something which I think will be obvious to everybody. You don't want to keep using the same needle in people. And this led very much to outbreaks of hepa-

titis. Since the 1940s, people have used evacuated blood collection tubes because they could automatically draw a predetermined blood volume and switching between tubes for additional samples pose a lower risk of spillage and needle stick injury and you use it all at once and throw it away. That's been the hope of this. So far good.

Glass evacuated tubes containing anticoagulants were commonly used from the 1950 s to the 1990s. Anticoagulants stopped the blood from coagulating, from thickening. You think this is easy. It's not easy. They started by using the wrong form of glass. They used something called soda lime glass and it would actually leach out things like calcium ions that would interfere with the tests they were doing. So they had to stop that. And then another thing happened. And this was more recent, at least in the United States. An organization called OSHA decided that it was unsafe to use glass in hospitals and clinics. Why? Because doctors and nurses would drop the blood-filled glass tubes; they would shatter, and then they would cut themselves on the glass and could become infected. So now in the USA OSHA has demanded that blood collection tubes be made from plastic. Plastic doesn't break when you drop it. That's of course good — like plastic milk bottles. The same switch has been taking place in many places. So that's what's happening.

They're manufactured through an injection molding using what are called polyesters. I'll show you what those are. These are polymers, most commonly being something called polyethylene terephthalate or PET. You've seen lots of bottles with PET as its name or combinations like that. And now what is it? Figure 3 shows the chemical structure of PET.

There is a linkage called a polyester linkage that connects the rigid terephthalate group with the flexible ethylene group. This is where an acid and the base would react to form an ester. Notice that this motif is repeated again and again; that's how you make polymers.

Polymers are an amazing thing. If it weren't for polymers, most of us would lose our clothes and be a bit embarrassed. So much is done with polymers today, including these tubes. So here's what's good about them. Compared to glass, plastic minimizes exposure to biohazardous materials following breakage. It has a greater shock resistance, it tolerates higher centrifugation speeds, people spin it around, centrifuge it. The plastic tube
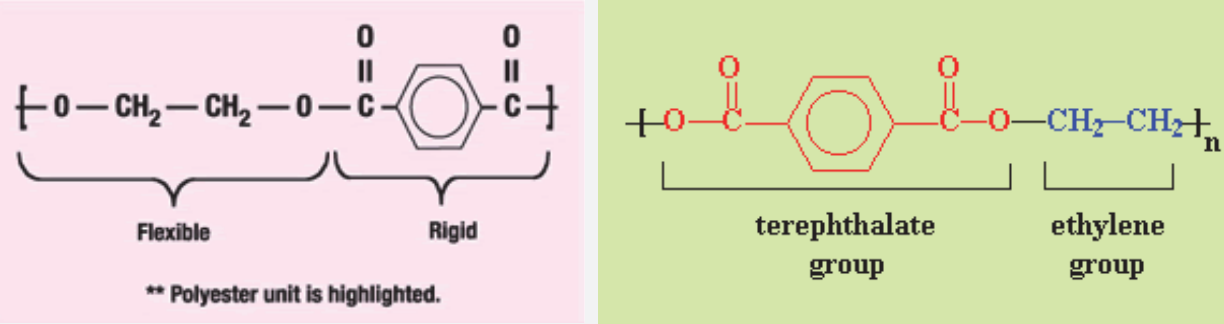

Figure 3. The PET family of plastics. The main characteristics of the PET family of plastics are their easy processing and resistance to impact and chemical agents as well as their transparency and ductility. 


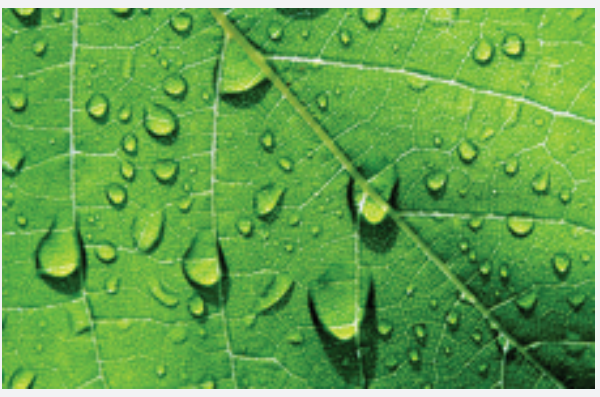

Figure 4. The surface of a leaf with morning dew condensed on it and balling up into droplets.

weighs less and has excellent dimensional precision, and is more easily disposed of by incineration at a lower cost. It is very hard to incinerate glass. This part works well.

Let me give you next a sense of what's bad, as pictured in Figure 4. Here you see water balling up on this leaf. This is because the leaf is waxy and is what we call hydrophobic, that is, hates water. And plastic tubes generally have a hydrophobic surface. They hate water and they do not efficiently activate the coagulation process. Clots formed on the plastic surface of tubes are gelatinous compared to clots formed in glass tubes. Blood does not flow smoothly. No one today uses bare plastic tubes. They are that bad: you have to fix this problem. They take out things from the blood and interfere with the tests.

So what does the manufacturer of blood collection tubes do? The fix to this problem is you spritz in, you squirt in, detergent; you add a surfactant coating to the inside of the blood collection tube. But these surfactants can leach out and interfere with the blood analysis. And this is why Dr. Bowen came to see me because they're causing him a lot of trouble. He says do something about this.

I'm going to show you what I think is relatively easy chemistry; in some sense, it looks like magic, but it's something that all of you could do if you want to and it shows you the power of chemistry. Here we have a surface that hates water. How are we going to make it so it likes water? It's a common problem. It's a problem that we all face about what causes things to dissolve other things. And generally the expression is that like likes like. You want to make something similar. So that to make it similar what we really want to do is add $-\mathrm{OH}$ groups. You all know that water is $\mathrm{H}_{2} \mathrm{O}$, that is, $\mathrm{H}-\mathrm{OH}$. Well, you want to put on the outside of the surface $-\mathrm{OH}$ groups called hydroxyl groups. That's what we want to do. And I'm going to show you how we do that.

Here is then a little bit of some chemistry, as shown in Figure 5. In organic chemistry, a nucleophilic addition reaction is an addition reaction where a chemical compound with an electron deficient or electrophilic double or triple bond, a $\pi$ bond, reacts with an electron rich reactant, termed a nucleophile. The magic word in chemistry is catalysis - to find something to speed things up which itself doesn't really play a role in what's happening. It intimately gets involved but it's recovered, it's recycled in the process. Look what happens. If you add, however, a strong acid to an alcohol, you're going to form an ester. This is not a good idea, this is the wrong approach. Instead a strong base is what you want and you want a strong base to catalyze the reaction by removing a proton from the alcohol and thus making it what we would call nucleophilic which makes if a better attacking group.

Now comes the next idea and it's an idea that's taken me a while to first realize. I didn't get this at all in high school. It just came up later. The idea is that you can actually find a base stronger than $\mathrm{OH}^{-}$, but the only way you can do that is not to work in water. Water, which is 55 molar, stops you. You must go to what's called a nonaqueous medium; then you can work with things called superbases. And superbases are extremely strong bases that have a higher affinity for protons than the $\mathrm{OH}^{-}$group. The hydroxide ion is the strongest base possible when you have water but bases exist with even much greater strength for protons and we're going to use these.

So here's our proposed fix. We plan to chemically convert the inner surface of the PET blood collection tube from hydrophobic to hydrophilic by adding an alcohol and a superbase in the absence of water. That's the goal I had in mind. Figure 6 shows you what we do.

The particular alcohol we added is called ethylene glycol, which is the common constituent of anti-freeze. At first, Sam Kim tried potassium hydroxide but later on we replaced it with superbases. They have names you can read in the caption to Figure 6 but l'm not going to bother to say them. In this trans-esterification process of PET we put $-\mathrm{OH}$ groups on the outside of the inner surface of the plastic blood collection tube. That's what we're doing. And that could be really neat. It turns out the chemistry is not original. It's been done before. Believe me it is not original at all. But the idea of making a new material, particularly a surface, appears to be original, and we have actually filed for a patent. 


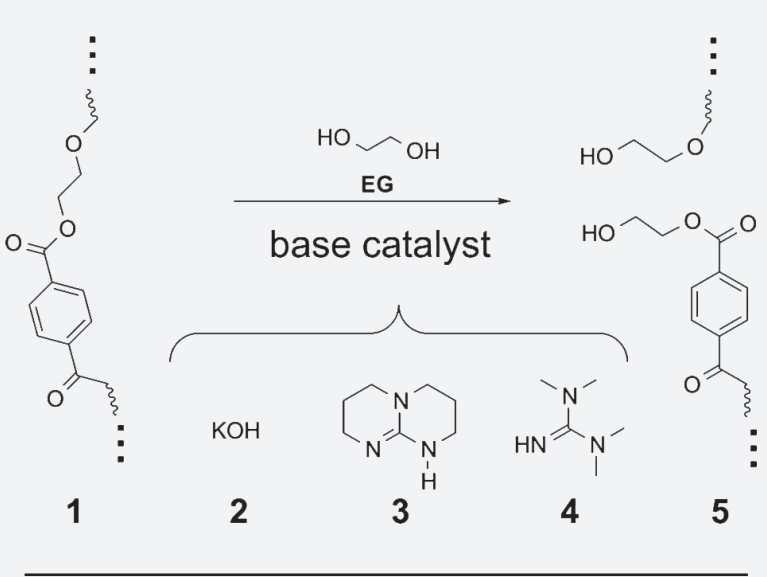

Figure 6. Trans-esterification reaction for transforming the surface property of PET (1) by contact with ethylene glycol (EG) in the presence of a base catalyst to a hydrophilic surface (5), which we call ChemoPET. The structures of three base catalysts we have used are shown: (2) potassium hydroxide (3) 1,5,7 triazabicyclo[4.4.0]dec-5-ene (TBD), and (4) 1,1,3,3-tetramethylguanidine (TMG).

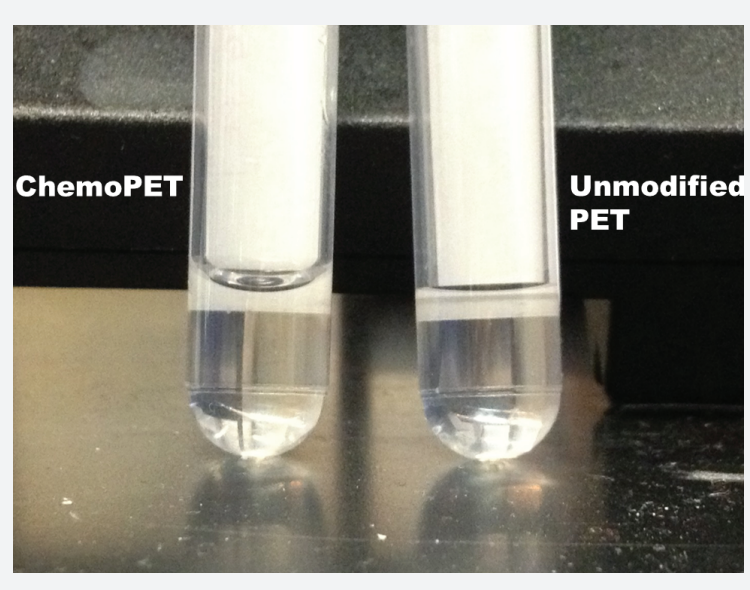

Figure 7. ChemoPET tube on the left and unmodified PET tube on the right, both filled with water.

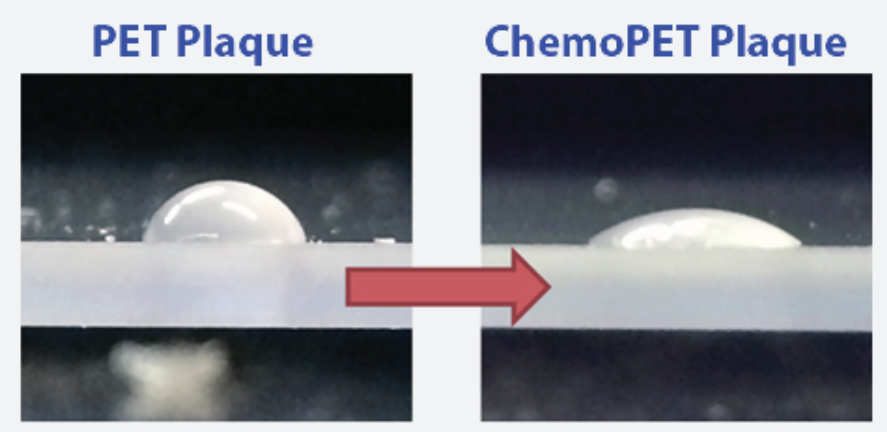

Figure 8. Water droplet on PET and ChemoPET plaques showing the spreading of the droplet on the latter surface.
So the question is of after this big preamble, does it work? Well, would I stand here before you if I did have some idea that it worked, but let me try to convince you that it does work. Perhaps, the best way to do that is to show you two test tubes made out of plastic, made out of PET (Figure 7).

Look at the PET blood collection tube that's unmodified. The water just doesn't like its hydrophobic surface and the meniscus is flat. Contrast this behavior with the ChemoPET blood collection tube whose surface has been made hydrophilic. The water meniscus bends upward because the water loves the hydrophilic surface. The surface of the ChemoPET blood collection tube is acting like the surface of a glass blood collection tube!

And I'm showing you before treatment and after treatment and I'm doing it on plaques of PET where you can actually again see how this water droplets spreads out. And it looks good, as shown in Figure 8.

What further excites me about this treatment process is it can be done in about ten minutes at room temperature, with essentially no loss of catalyst and hardly any consumption of the ethylene glycol because this is a surface modification. It also has an outstanding shelf life. Consequently, the making of ChemoPET tubes from PET tubes looks to be inexpensive, quick, and not very laborious. Only time will tell whether this can supplant existing blood collection tubes made by an established and entrenched technology. We have been assessing its performance in blood collection analysis and it seems to be comparable to the use of glass blood collection tubes. More detailed comparisons have been published after this presentation was made:

S. Kim, R. A. R. Bowen, and R. N. Zare, "Transforming Plastic Surfaces with Electrophilic Backbones from Hydrophobic to Hydrophilic," ACS Applied Materials \& Interfaces 7, 1925-1931 (2015).

R. A. R. Bowen, S. C. Kim, A. Sattayapiwat, V. AustriaEsguerra, and R. N. Zare, "Performance of Chemically Modified Plastic Blood Collection Tubes," Clin. Biochem. 49, 90-99 (2016).

I hope this short presentation has given you an idea of this research field. It's one example of the power of chemical transformations and it shows what you're able to do with a little bit of chemistry understanding to really change the nature of materials. Yes, it's magic. But it's magic that we understand and can control and do much more with. There's going to be yet more exciting developments, I believe, presented to you today that will impact our lives. Here's a very simple one. And I thank you to have this opportunity to present this to you. 
[Applause]

Host: Thank you Dick. Apologies for believing that Bengt actually was trying to impersonate John Cleese when I say that, no, he really slipped.

Richard Zare: He was attempting to fly.

Host: Yeah, so unfortunately he had to go to the hospital. So he won't be here today. So to chair this session instead, we have Magdalena Eriksson, who is also on the Board of Molecular Frontiers.

Magdalena Eriksson: Good morning everybody. It's great to see this room full of people even upstairs. So I think Bengt's introduction of Dick was a little bit interrupted, but you can see Dick is an enormously enthusiastic person who works in different areas of chemistry and this is one exciting example of that. So if you have any questions, feel free to raise your hand and the microphones will come from the side or the aisles of the room. Who's first?

Richard Zare: Any thoughts about this?

Magdalena Eriksson: Do you ever wonder what happens to your blood?

Audience: So you said that the important thing is that the blood doesn't interact with the material. So what does it matter? Does the material like water or not, does it stick to it or not? What is it?

Richard Zare: So you see that it really does like water and it's not causing the things which don't like water, things that are called like fibrinogen and other things that stick to the tube. It's making it so it can easily be smooth and doesn't stick any further. As you know, most biological fluids contain water, right? And we don't want to take things out, you know most of us are all wet, almost all wet, right? And that's what you want. So it doesn't interfere with the system. That's its nature. We didn't know ahead of time how well this would work. And I'm just giving you a sense of something about research, if I might. Almost all the time everything in our lab doesn't work. We fail, go through stages of failure. And it's very important because it's not like what you experience in high school. In high school they design labs that always work, are supposed to work even those don't work so well but they are supposed to work. In real research, things don't work often and you have to be patient and persistent and you have to let failure guide you - honestly — to make things work. That's an important part if you want to be a researcher, but it's immensely rewarding. Those moments are so exciting when something starts to happen. So some of us can't stop doing it. It's a passion.

Magdalena Eriksson: Any arms raised? No. You know I don't want to challenge you, but in Singapore you can't even keep up with all the arms the people raise, so many. So let me ask you Dick, what happens when you get an answer to a question. How many more questions do you normally start asking.

Richard Zare: So there are further questions we don't know the answers to. What happens at various sites that we really modify and are there others that we do not modify. I don't know fully the answer to that. Need to try to find that out. Maybe there's some way of titrating it or something to find out. We've done other techniques or some very fantastic forms of microscopy that today one can have. So far we've used like atomic force field but we haven't learned anything from it, we're trying but it's really challenging. One of the amazing things that's happened today from the time when I was your age till now is that people can look at individual molecules, okay. And really believe, that we prided ourselves that we believed in them but no one had seen one. That's all changed. You can see them a number different ways.

Magdalena Eriksson: And there will be a discussion.

Richard Zare: Question over there.

Magdalena Eriksson: Great.

Audience: How can you stop blood from coagulating?

Richard Zare: They actually add materials that are anticoagulants. Now I don't know enough of the chemistry behind that to know how that slows that down, but I know there are such materials. Heparin I think is such a material. But I don't know fully how it works. Maybe someone else does. But there are such materials that people use regularly. And you know there are so many tests we now run with blood to try to figure out what's with us and it's important. Dipstick, okay, like we do a dipstick into an engine and look at the oil, dirty or not, and determine how the engine is. Well, we look at blood, we try to learn from that and other bodily secretions and to get a quick idea of what's happening with us.

Audience: You asked is this approach universal. And you don't answer your own question. To continue, my question is have you considered changing not only the chemistry or the material but also the morphology of the surface of the material that were influenced very strongly the hydrophobic, hydrophilic properties of it.

Richard Zare: I have not done much but I know that in general a rough surface is worse than a smooth surface. I hope you agree with me.

Audience: Worst or good in the sense...

Richard Zare: Yeah I think a rough surface has more surface area and can generally pick things up more. So these tend to be smooth surfaces that are being modified, but I don't know if that's what you meant by morphology. Maybe you meant something else. And it may not be the only way but plastics 
are really cheap and it's easy to work with and this looks like a simple fix to a problem. And so I hope people might take this up. We'll see.

Audience: I wonder this technique, is this possible to use like in other things in daily life, have you thought about that? Like, yeah hating and love water, if you can use it like in other things except this blood thing.

Richard Zare: If I don't understand the question...

Magdalena Eriksson: Can you please ask the question again.

Audience: Yes. This technique that you have used to discover what you have discovered, is it possible to use the technique to other things of your daily life.
Richard Zare: Okay, so far I think it's general to what we call polyesters, to the things that have nucleophilic backbones to do this type of chemistry. But the idea of surface modifications is a big field. And this is just one example of what I think many people are doing or can do.

Magdalena Eriksson: Yeah, well, I think we move on and you come back for the panel discussion in the afternoon. Thank you very much, Dick. Can we give him a round of applause.

[Applause] 